\title{
Long noncoding RNA MALAT1 regulates sepsis in patients with burns by modulating miR-214 with TLR5
}

\author{
FENGYING GAO $^{1}$, RONG CHEN $^{1}$, YAOFENG XI ${ }^{1}$, QIUJIAN ZHAO ${ }^{2}$ and HAIFENG GAO ${ }^{2}$ \\ ${ }^{1}$ Department of Plastic and Burn; ${ }^{2}$ Clinical Laboratory, Baoji Center Hospital, Baoji, Shaanxi 721008, P.R. China
}

Received April 8, 2017; Accepted January 21, 2019

DOI: $10.3892 / \mathrm{mmr} .2019 .10028$

\begin{abstract}
The present study aimed to identify the involvement of the dysregulation of the metastasis-associated lung adenocarcinoma transcript-1 (MALAT1)/microRNA (miR)-214/Toll-like receptor (TLR)5 signaling pathway in the development of post-burn sepsis. THP-1 cells were used in the present study, in addition to 8-10 week-old mice. ELISA analysis was performed to examine the expression levels of inflammation-associated factors. Reverse transcription-quantitative polymerase chain reaction (RT-qPCR) analysis and western blotting were performed to analyze the influence of burns or burns with infection on the production of MALAT1, miR-214 and TLR5. Commonly-used software and a luciferase assay was used to confirm the target gene of miR-214. RT-qPCR analysis and western blotting were performed to elucidate the effects of lipopolysaccharide (LPS), miR-214 and MALAT1 on the expression of miR-214, TLR5, tumor necrosis factor (TNF)- $\alpha$, interleukin (IL)- 6 and IL-10. Burn injury increased TLR5, TNF- $\alpha$, IL-6 and IL-10 expression levels, which were abolished by treatment with MALAT1. miR-214 directly targeted TLR 5 by binding to the TLR 5 3' untranslated region (ITR), and the luciferase activity of the wild-type, and not the mutant, TLR5 3'UTR was reduced following transfection with miR-214. In cells not treated with LPS, MALAT1 and anti-miR-214 significantly enhanced TLR5, TNF- $\alpha$, IL-6 and IL-10 expression, and repressed miR-214 production; whereas, miR-214 and MALAT1 short hairpin (sh)RNA decreased TLR5, TNF- $\alpha$, IL-6 and IL-10 expression levels, and increased miR-214 expression. In cells treated with LPS, LPS reduced miR-214 expression and increased TLR5, TNF- $\alpha$, IL-6 and IL-10 expression compared with LPS-untreated cells, and the effects of MALAT1, anti-miR-214, miR-214 and MALAT1 shRNA on TLR5, TNF- $\alpha$, IL-6 and IL-10 were the same as in LPS-untreated cell. The results of the present
\end{abstract}

Correspondence to: Dr Haifeng Gao, Clinical Laboratory, Baoji Center Hospital, 8 Jiangtan Road, Baoji, Shaanxi 721008, P.R. China E-mail: malatburn@163.com

Key words: metastasis-associated lung adenocarcinoma transcript-1, sepsis, microRNA-214, Toll-like receptor 5, burn, monocytes study indicated the association between the dysregulation of MALAT1/miR-214/TLR5 and the risk of post-burn sepsis.

\section{Introduction}

Serious burn trauma is destructive damage associated with a hyper-metabolic and catabolic state. Furthermore, it compromises the immune system by activating systemic inflammation, frequently leading to suboptimal innate immunity (1). Damage to the skin barrier and alterations in immunity elevate the susceptibility of patients with burns to infections in the burn wound, and may cause systemic bacterial spread, triggering multi-organ failure, sepsis and mortality (1). A variety of patient factors, including depth of burn, extent of injury, immune status and age, combined with microbial factors, including toxin production, enzymes, number and type of organisms and mobility, are determinants of the possibility of aggressive burn wound infection. Burns with a large surface area are associated with infections in the burn wound leading to multi-organ failure, sepsis and mortality $(2,3)$. Infection is a frequent trigger of complications in patients with serious burns. Burns trigger damage to the barrier that protects the skin. This provides an optimal opportunity for microbial colonization and likely spread into the blood and other organs, which may cause sepsis. Therefore, infection is the principal factor leading to mortality in patients with burns who survive the early phase of burn shock (4). Williams et al (5) performed a clinical observational study and demonstrated that sepsis accounted for $47 \%$ of the mortality in patients with burns at a large pediatric burn center. The study of Mann et al (6) reported that patients with burns have a high incidence of sepsis and associated unfavorable outcomes.

The majority of transcribed RNAs are non-coding in mammalian cells (7). Numerous RNAs produce small RNAs, including microRNAs (miRs), a well-known family of small RNAs, in addition to small nucleolar RNAs, tRNA-derived stress-induced RNAs, transcription initiation RNAs, Piwi-interacting RNAs and small interfering RNAs (8). Other transcripts [long non-coding RNAs (lncRNAs)] consist of $>200$ nucleotides (9). ncRNAs impact the modulation of genes at all levels via their interactions with proteins, RNA and DNA, including protein stability, mRNA translation, mRNA turnover, pre-mRNA splicing, transcription and chromatin remodeling (10). 
A previous study demonstrated that metastasis-associated lung adenocarcinoma transcript-1 (MALAT1) has a regulatory role in the generation of inflammatory cytokines in certain human cancers, including lung, liver, breast, cervical and bladder cancers (11). Yan et al (12) first demonstrated the alteration in MALAT1 expression in the retina in patients with diabetes. It has been demonstrated that the glucose-mediated upregulation of the inflammatory mediators tumor necrosis factor (TNF)- $\alpha$ and interleukin (IL)- 6 is modulated by lncRNA MALAT1 via stimulation of serum amyloid A3 (SAA3); it was identified that early elevation, followed by a reduction in MALAT1, positively increases the expression of inflammatory ligands, including SAA3, finally enhancing the expression of inflammatory cytokines, including TNF- $\alpha$ and IL-6 (13). Further duration-dependent studies at different phases of diabetes using animal models (long-term, short-term and acute) may indicate whether this IncRNA, MALAT1, is an actual initiator of oxidative stress and inflammation (13). The flagellin receptor Toll-like receptor 5 (TLR5) is among the most frequently-triggered pattern recognition receptors (14). Allelic variations in TLR5 may make infants prone to sepsis, and high-level production of TLR5 in septic individuals is positively associated with more serious disease $(14,15)$. Using computational analysis, TLR5 was identified to be a potential target gene of miR-214 in the present study, and TLR5 has been reported to be functionally involved in the pathogenesis of post-burn sepsis; the occurrence of sepsis is important for the prognosis of patients with burns $(16,17)$. The present study analyzed the association between the dysregulation of MALAT1, miR-214 or TLR5 and the risk of post-burn sepsis.

\section{Materials and methods}

Mouse model of burn injury and burn wound infection. The Institutional Animal Care and Use Committee of Baoji Center Hospital (Baoji, China) approved all procedures involving animals. A total of 48 female C57BL/6 mice at 8-10 weeks of age (weight, $21.2 \pm 2.8 \mathrm{~g}$ ) were obtained from the Animal Center of Baoji Center Hospital and used in the present study. Mice were housed in the appropriate institutional animal care facility, and acclimatized for 7 days post-arrival at $25^{\circ} \mathrm{C}$ with $60 \%$ humidity. The acclimatization was performed under a 12/12 light/dark cycle; mice were provided food and water ad libitum. A well-established mouse model with full-thickness cutaneous burn injury was utilized in the present study. Buprenorphine $(0.1 \mathrm{mg} / \mathrm{kg})$ was utilized to treat the mice subcutaneously for $30 \mathrm{~min}$ for analgesia prior to the burn injury. Isoflurane $(2.5 \%)$ was used for general anesthesia. The dorsal surface of the mouse was shaved, and $1 \mathrm{ml}$ normal saline was used to treat the mice subcutaneously to avoid injury to the underlying tissues. A $35 \%$ total surface area scald burn was caused by exposing the shaved dorsal area to $97-98^{\circ} \mathrm{C}$ water for $10 \mathrm{sec}$ using a molded template with a rectangular opening. A total of $2 \mathrm{ml}$ Ringer's lactate solution (Kelun Pharmaceutical, Chengdu, China) was utilized to treat the mice immediately for fluid resuscitation via the intraperitoneal route, and buprenorphine $(0.1 \mathrm{mg} / \mathrm{kg})$ was used to treat the mice secondarily post-burn procedure. Burn-injured mice were housed in sterile cages individually, and allowed food and sterile water. Burn wounds were collected for analysis under anesthesia. Pseudomonas aeruginosa bacteria were seeded on the surface of the burn wound. A total of $1 \times 10^{8}$ colony-forming units/ml (200 $\mu \mathrm{l})$ Pseudomonas aeruginosa were incubated on the surface of the burn wound 4 days post-burn to cause wound infection. A hematology analyzer was used to determine blood cell count of the animal groups.

Cell culture and transfection. THP-1 cells were obtained from the Chinese Cell Bank of the Chinese Academy of Sciences (Shanghai, China). RPMI medium (Gibco; Thermo Fisher Scientific, Inc., Waltham, MA, USA) containing 10\% heat-inactivated fetal bovine serum (Life Technologies; Thermo Fisher Scientific, Inc.), $100 \mathrm{mg} / \mathrm{ml}$ streptomycin, $50 \mathrm{U} / \mathrm{ml}$ penicillin and $2 \mathrm{mM}$ glutamine (Euroclone Diagnostica S.r.l., Siziano, Italy) was used to maintain the THP-1 cells, in a humidified incubator with $5 \% \mathrm{CO}_{2}$ at $37^{\circ} \mathrm{C}$. In order to perform cell transfections, THP-1 cells were cultured in 24-well plates at a concentration of $2 \times 10^{3}$ cells per well, and Attractene Transfection Reagent (Qiagen $\mathrm{GmbH}$, Hilden, Germany; cat. no. 1051531) was used to transfect $15 \mathrm{pmol} / \mathrm{ml}$ miR-675-5p mimic (Life Technologies; Thermo Fisher Scientific, Inc.; cat. no. 4464066) or miR-675-5p inhibitor (Life Technologies; Thermo Fisher Scientific, Inc.; cat. no. 4464084) or scramble control (miR-NC 5'-CTA AGGTTAAGTCGCCCTCGCT-3') or $15 \mathrm{pmol} / \mathrm{ml}$ MALAT1 shRNA into THP-1 cells. Each reaction was performed in triplicate; analysis was conducted $48 \mathrm{~h}$ following transfection.

THP-1 cell polarization and lipopolysaccharide (LPS) treatment. A total of $20 \mathrm{ng} / \mathrm{ml}$ interferon (IFN) $-\gamma$ (PeproTech, Inc., Rocky Hill, NJ, USA) with 10 ng/ml LPS (Sigma-Aldrich, St. Louis, MO) was used to direct PMA-differentiated THP-1 cells to the M1 phenotype, and $20 \mathrm{ng} / \mathrm{ml}$ IL-4 (PeproTech, Inc.) and $20 \mathrm{ng} / \mathrm{ml} \mathrm{IL-13} \mathrm{(Sigma-Aldrich;} \mathrm{Merck} \mathrm{KGaA,}$ Darmstadt, Germany) was used to direct PMA-differentiated THP-1 cells to the M2 phenotype. THP-1 cells were seeded into 96 -well plates at a concentration of $2 \times 10^{3}$ cells per well, and exposure to polarizing conditions was performed. Reverse transcription-quantitative polymerase chain reaction (RT-qPCR) analysis was performed to identify the polarized phenotypes. A total of $1 \mu \mathrm{g} / \mathrm{ml}$ LPS was used to treat THP-1 cells for $24 \mathrm{~h}$ when the cells had grown to $80 \%$ confluence.

RNA isolation and RT-qPCR. TRIzol reagent (RNAprep Pure Cell/Bacteria kit; Tiangen Biotech Co., Ltd., Beijing, China) was used to isolate the total RNA from THP-1 cells and peripheral blood mononuclear cells (PBMCs) from blood samples collected from the experimental mice, in accordance with the manufacturer's protocol. A PrimeScript ${ }^{\mathrm{TM}}$ RT Reagent kit (Perfect Real-Time; Takara Bio, Inc., Otsu, Japan) was used to perform reverse transcription to obtain cDNA according to the manufacturer's protocols, using $2 \mu \mathrm{g}$ cell-extracted RNA or $300 \mathrm{ng}$ total tissue RNA from the burn wound as the template. LightCycler 480 SYBR Green I Master (Roche, Basel, Switzerland) was used to perform qPCR analysis to determine the relative expression of lncMALAT1, miR-214 and TLR5. The qPCR cycling conditions was as follows: Initial polymerase activation at $95^{\circ} \mathrm{C}$ for $10 \mathrm{~min}$, followed by 40 cycles for $15 \mathrm{sec}$ at $95^{\circ} \mathrm{C}$ and $60 \mathrm{sec}$ at $60^{\circ} \mathrm{C}$. The expression of TLR5 mRNA (F: 5'-CTTCTTGGGCGGCAATAAAC-3'; 
R: 5'-CCACTAAAGCATAAGTAGGCATC-3';), IncMALAT1 (F: 5'-GGTAACGATGGTGTCGAGGTC-3'; R: 5'-CCAGCA TTACAGTTCTTGAACATG-3';) and miR-214 (F: 5'-TTT CCTATGCATATACTTCTTT-3'; R: 5'-CAGTGCGTGTCG TGGAGT-3';) was calculated relative to the expression level of the endogenous controls $\beta$-actin (F: 5'-ACTCGTCATACT CCTGCT-3'; R: 5'-GAAACTACCTTCAACTCC-3';) and U6 (F: 5'-CTCGCTTCGGCAGCACA-3'; R: 5'-AACGCTTCA CGAATTTGCGT-3';), respectively. The value of $2^{-\Delta \Delta C q}(18)$ was used to describe the relative gene expression level of TLR5 mRNA and miR-214. Each experiment was performed at least three times.

Luciferase assay. Target gene prediction was conducted between TRAF3 and miR-214 with online themiRNA database TargetScan release 7.2 (www.targetscan.org). The segment of the wild-type TLR5 3' untranslated region (UTR) containing the putative binding site of miR-214 was synthesized and amplified using PCR with Applied Biosystems ${ }^{\circledR}$ TaqMan ${ }^{\circledR}$ MicroRNA Reverse Transcription Kit (Thermo Fisher Scientific, Inc.), and the PCR products were cloned into the plasmid PGL3-basic (Invitrogen; Thermo Fisher Scientific, Inc.). A $25 \mu 1$ reaction system was at utilized with $5 \mathrm{U}$ Taq Polymerase (Thermo Fisher Scientific, Inc.). The sequences of the primers used for PCR were as follows: TLR5 forward, 5'-GCTGCAACTGGACCTTTC G-3' and reverse, 5'-CCCAAACAGTCGAGGATTCAA-3'. The temperature protocol was as follows: $95^{\circ} \mathrm{C}$ for $3 \mathrm{~min}$, followed by 30 cycles of $94^{\circ} \mathrm{C}$ for $40 \mathrm{sec}, 56^{\circ} \mathrm{C}$ for $35 \mathrm{sec}$ and final extension at $72^{\circ} \mathrm{C}$ for $60 \mathrm{sec}$. The binding site of miR-214 located in the TLR5 3'UTR was mutated using a QuikChange XL mutagenesis kit (Stratagene; Agilent Technologies, Inc., Santa Clara, CA, USA) to generate a mutant TLR5 3'UTR. The mutant TLR5 3'UTR was cloned into the plasmid PGL3-basic (Invitrogen; Thermo Fisher Scientific, Inc.). Lipofectamine ${ }^{\circledR} 2000$ (Invitrogen; Thermo Fisher Scientific, Inc.) was used to co-transfect the $2 \times 10^{3}$ cells per cell THP-1 cells with $0.02 \mu \mathrm{g}$ control vector containing Renilla luciferase (Promega Corporation, Madison, WI, USA) or $0.4 \mu \mathrm{g}$ firefly luciferase reporter vector containing the wild-type or mutant TLR5 3'UTR, and miR-214 mimic or miR-NC. After $12 \mathrm{~h}$ post-transfection, a dual-luciferase reporter system (Promega Corporation) was used to detect the luciferase activity of Renilla and firefly luciferase, according to the manufacturer's protocol. All tests were performed three times.

Western blot analysis. PBS was used to wash the cells and PBMCs twice, and radioimmunoprecipitation assay buffer (Thermo Fisher Scientific, Inc.) was used to lyse the THP-1 cells and PBMCs on ice for $10 \mathrm{~min}$. A bicinchoninic acid (BCA) assay (Thermo Fisher Scientific, Inc.) was used to measure the concentration of protein, according to the manufacturer's protocol. Loading buffer was used to resuspend the protein fractions, which were denatured for $10 \mathrm{~min}$ at $100^{\circ} \mathrm{C}$. SDS-PAGE on a $10 \%$ gel was used to separate $20 \mu \mathrm{g} /$ lane proteins, which were subsequently transferred to polyvinylidene fluoride membranes (EMD Millipore, Billerica, MA, USA). TBS-Tween 20 buffer (0.1\% Tween 20$)$ with $5 \%$ fat free milk was used to block the membrane at room temperature for $120 \mathrm{~min}$. The primary rabbit polyclonal antibody against TLR5 at a dilution of 1:5,000 (cat. no. DPAB-DC3077, Upstate Biotechnology, Inc., Lake Placid, NY, USA) and anti- $\beta$-actin antibody at a dilution of 1:12,000 (cat. no. 4970S, Cell Signaling Technology, Inc., Danvers, MA, USA) were utilized to incubate the membrane at $4^{\circ} \mathrm{C}$ for $12 \mathrm{~h}$. Subsequently, horseradish peroxidase-conjugated secondary antibody at a dilution of 1:15,000 (cat. no. AIgYFc-HRP, Upstate Biotechnology, Inc.) was used to treat the membrane for $2 \mathrm{~h}$ at room temperature. Chemiluminescence (Amersham; GE Healthcare, Chicago, IL, USA) was used to detect the bands of protein. A total of three independent experiments were performed.

ELISA analysis. Radioimmunoprecipitation assay buffer (Thermo Fisher Scientific, Inc.) was used to extract the protein from THP-1 cells and PBMCs, and the BCA assay (Thermo Fisher Scientific, Inc.) was used to measure the concentration of protein. Total protein lysates were isolated from same treatment groups, and these lysates were also subjected to ELISA assay. Human SAA3 (cat no. CSB-E11836h, Cusabio Biotech Co., Ltd., Wuhan, China) and ELISA kits were used to detect the expression levels of TLR5, TNF- $\alpha$ (cat. no. DTA00C, R\&D Systems, Inc., Minneapolis, MN, USA), IL-6 (cat. no. D6050, R\&D Systems, Inc., Minneapolis, MN, USA) and IL-10 (cat. no. D1000B, R\&D Systems, Inc., Minneapolis, MN, USA) based on the absorbance at $450 \mathrm{~nm}$, which was normalized to the background absorbance at $568 \mathrm{~nm}$. A total of three independent experiments were repeated.

Statistical analysis. All data are presented as the mean \pm standard error of the mean. SPSS 19.0 statistical software (IBM Corp., Armonk, NY, USA) was used to perform the statistical analysis. An unpaired t-test was used to evaluate the statistical significance of comparisons between two groups. Two-way analysis of variance and the Student-Newman-Keuls multiple comparison test were used to evaluate the statistical significance of comparisons among more than two groups. $\mathrm{P}<0.05$ was considered to indicate a statistically significant difference. All experiments were repeated three times.

\section{Results}

Elevation of pro-inflammatory cytokines caused by burn injury or post-burn infection is attenuated by treatment with MALAT1. The present study established animal models of burn injury and post-burn infection, and it was identified that burn injury caused a significant decrease in the white blood cell count compared with the sham controls (Fig. 1A) using a hematology analyzer. In addition, post-burn infection caused a further decrease in white blood cell count compared with the sham and burn groups, which was eliminated by treatment with short hairpin (sh)RNA-MALAT1 (shMALAT1) (Fig. 1A). Burn injury caused a significant increase in TNF- $\alpha$ (Fig. 1B), IL-6 (Fig. 1C) and IL-10 (Fig. 1D), and post-burn infection caused a further increase in TNF- $\alpha$ (Fig. 1B), IL-6 (Fig. 1C) and IL-10 (Fig. 1D) compared with the burn groups, which was abrogated by treatment with shMALAT1, indicating that the burn injury and post-burn sepsis-induced inflammatory reaction may be attenuated by shMALAT1.

MALAT1, miR-214 and TLR5 are associated with burn injury and post-burn sepsis. An evident increase in MALAT1 mRNA (Fig. 2A) expression levels was observed in the burn group 

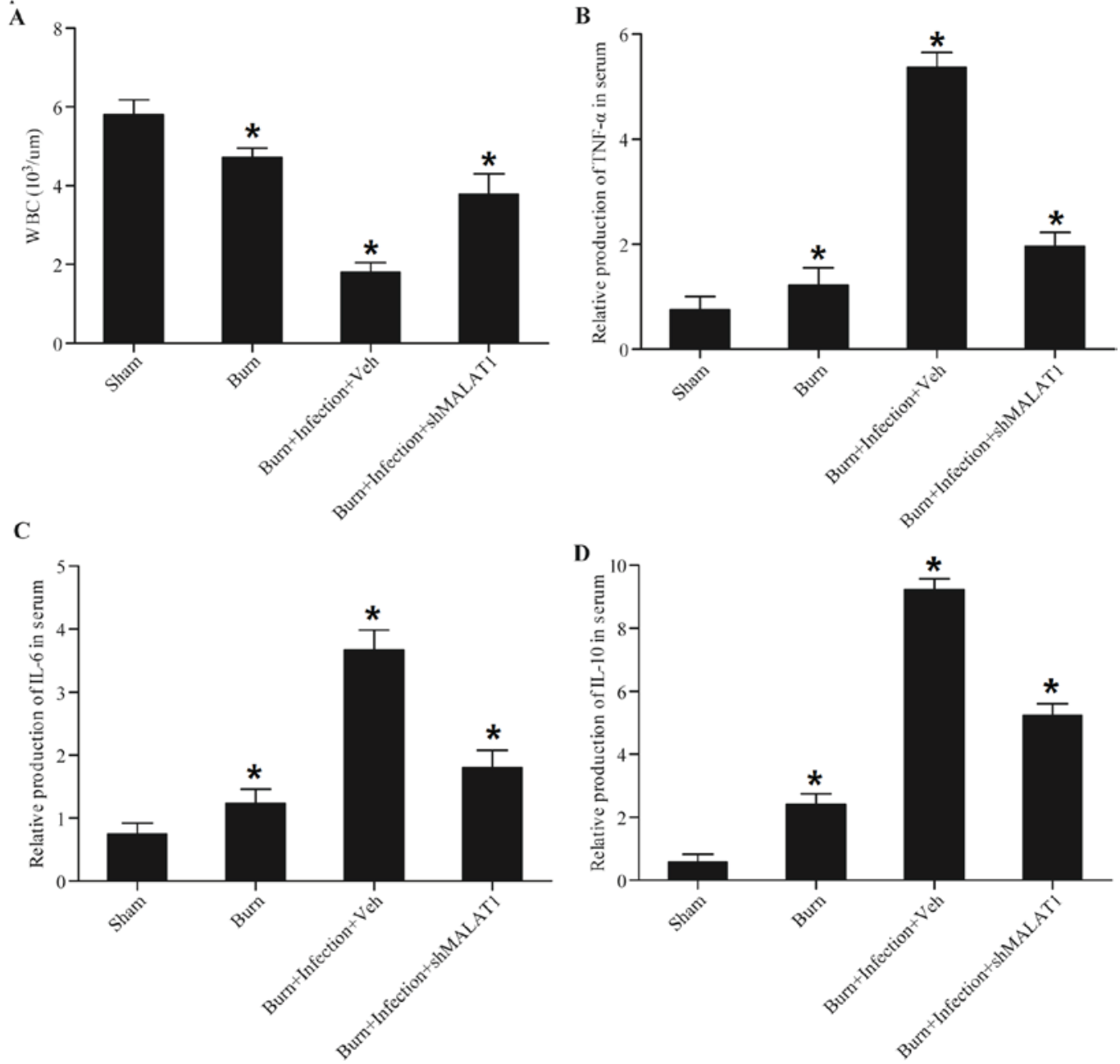

Figure 1. Elevation of proinflammatory cytokines caused by burn injury or post-burn infection is attenuated by treatment with MALAT1. A mouse model of burn injury and burn wound infection was established, peripheral blood samples were collected from all mice and monocytes were isolated from the blood samples. WBC count, TNF- $\alpha$, IL-6 and IL-10 were detected. Burn injury induced a decrease in (A) WBC count, and post-burn infection caused a greater decrease in WBC count, which was abolished following transfection with shMALAT1. Burn injury induced an increase in (B) TNF- $\alpha$, (C) IL-6 and (D) IL-10, and post-burn infection caused a greater increase in TNF- $\alpha$, IL- 6 and IL-10 expression, which was abolished by transfection with shMALAT1. " $\mathrm{P}<0.01$ vs. sham. Veh, vehicle; sh, short hairpin; TNF- $\alpha$, tumor necrosis factor- $\alpha$; IL, interleukin; MALAT1, metastasis-associated lung adenocarcinoma transcript-1; WBC, white blood cell.
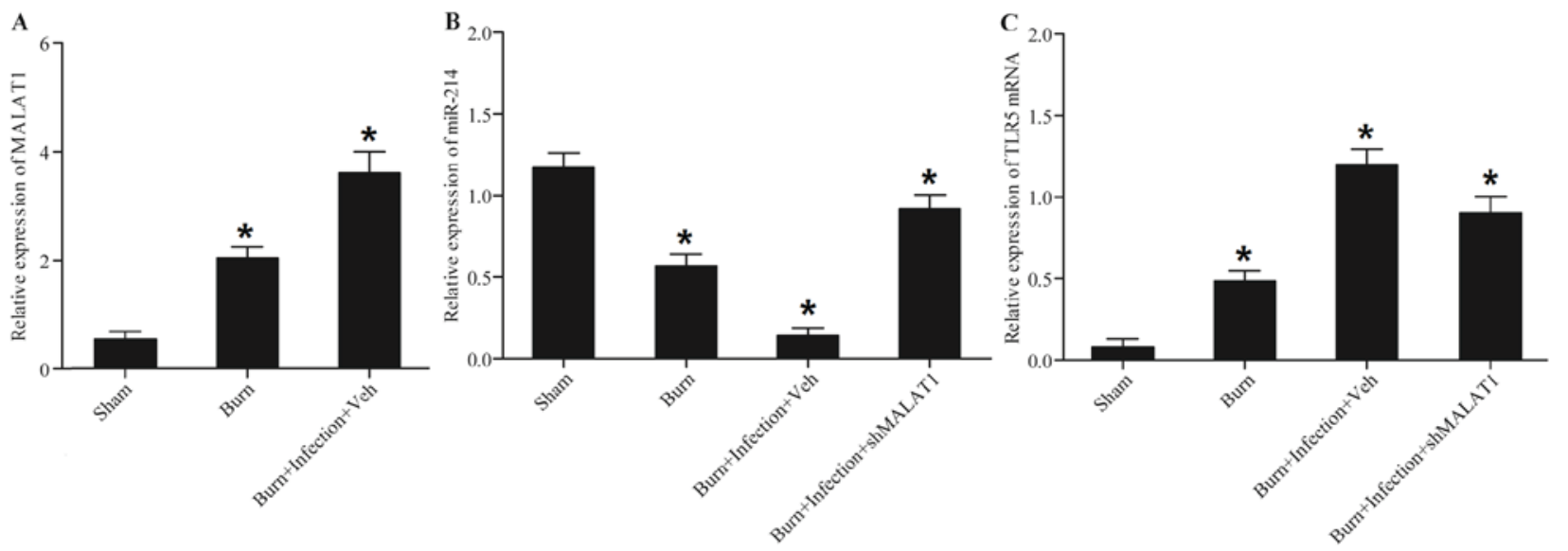

Figure 2. MALAT1, miR-214 and TLR5 are associated with burn injury and post-burn sepsis. Peripheral blood samples were collected from all mice, and monocytes were isolated from the above blood samples. MALAT1, miR-214 and TLR5 were detected in the monocytes. (A) The burn and post-burn infection groups exhibited significantly upregulated MALAT1 expression. (B) Burn injury inhibited miR-214 expression, and the inhibitory effect on miR-214 expression was greater in the burn plus infection group; however, treatment with shMALAT1 restored miR-214 expression. (C) Burn injury promoted TLR5 expression, and the promoting effect on TLR5 expression was greater in the burn plus infection group; however, the effect was abrogated by shMALAT1. ${ }^{\text {"P }}<0.01$ vs. sham. miR, microRNA; TLR5, Toll-like receptor 5; sh, short hairpin; MALAT1, metastasis-associated lung adenocarcinoma transcript-1. 
compared with the sham control; post-burn infection caused a further increase in its expression. By contrast, a significant decrease in miR-214 expression (Fig. 2B) was observed in the burn group compared with the sham control, and post-burn infection further enhanced this decrease, which was attenuated by treatment with shMALAT1. The results for TLR5 expression (Fig. 2C) were comparable to those for MALAT1. The increase in the expression of TLR5 was attenuated by treatment with shMALAT1.

TLR5 is a candidate target gene of miR-214. TLR5 and TNF receptor-associated factor 3 (TRAF3; Fig. 3) were predicted to be target genes of miR-214 using online miRNA databases, including TargetScan. The putative binding sequences of miR-214 in the 3'-UTRs of TLR5 and TRAF3 are conserved among different species. To substantiate whether miR214 targets TLR5 and TRAF3 directly, luciferase reporter vectors were constructed containing a wildtype or mutant 3'-UTR of TLR5 and TRAF3, in which the sequences corresponding to the seed sequences were altered. As presented in Fig. 3B, miR-214 mimics significantly reduced the luciferase activity of wild-type TLR5 compared with the control, whereas the luciferase activity of mutant TLR5 (Fig. 3B), wild-type and mutant TRAF3 (Fig. 3D) in miR-214-overexpressing cells exhibited no significant difference, indicating that TLR5 is a virtual target gene of miR-214.

MALAT1 and miR-214 alter the production of inflammationassociated factors. LPS is considered to be a major toxin in sepsis, and is the primary cause of the enhanced production of pro-inflammatory cytokines that is observed in sepsis (19). The expression levels of miR-214, TLR5, TNF- $\alpha$, IL- 6 and IL-10 were assessed in LPS-treated or untreated THP-1 cells transfected with MALAT1, anti-miR-214, MALAT1 shRNA and miR-214. As presented in Fig. 4, transfecting with MALAT1 and anti-miR-214 reduced miR-214 expression (Fig. 4A), while enhancing TLR5 mRNA (Fig. 4B) and protein (Fig. 4C), TNF- $\alpha$ (Fig. 4D), IL-6 (Fig. 4E) and IL-10 (Fig. 4F) expression compared with the scramble control.

Cells treated with LPS exhibited a lower level of miR-214 (Fig. 5A) compared with that in untreated cells. Furthermore, MALAT1 and anti-miR-214 induced a further decrease in the miR-214 expression level compared with the scramble control. By contrast, cells treated with LPS displayed higher levels of TLR5 mRNA (Fig. 5B) and protein (Fig. 5C), TNF- $\alpha$ (Fig. 5D), IL-6 (Fig. 5E) and IL-10 (Fig. 5F) compared with untreated cells. Furthermore, treatment with MALAT1 and anti-miR-214 induced a decrease in TLR5 mRNA (Fig. 5B) and protein (Fig. 5C), TNF- $\alpha$ (Fig. 5D), IL-6 (Fig. 5E) and IL-10 (Fig. 5F) expression levels compared with the scramble control. Simultaneously, MALAT1 shRNA and miR-214 significantly promoted the relative expression of miR-214 (Fig. 6A) compared with the scramble control. MALAT1 shRNA and miR-214 significantly repressed TLR5 mRNA and protein expression (Fig. 6B), and transfection of MALAT1 shRNA and miR-214 significantly downregulated the expression of MALAT1 (Fig. 6C), TNF- $\alpha$ (Fig. 6D), IL-6 (Fig. 6E) and IL-10 (Fig. 6F) compared with the scramble control.
A

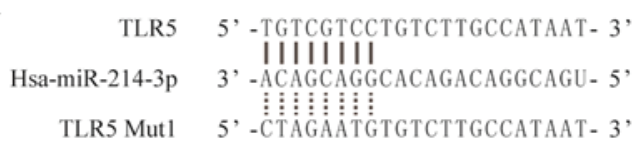

B

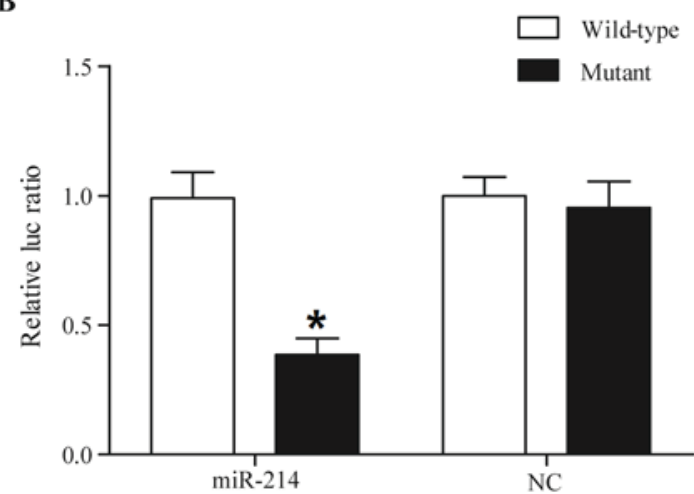

C

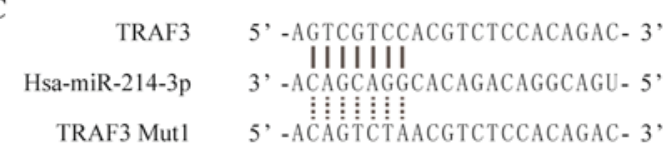

D

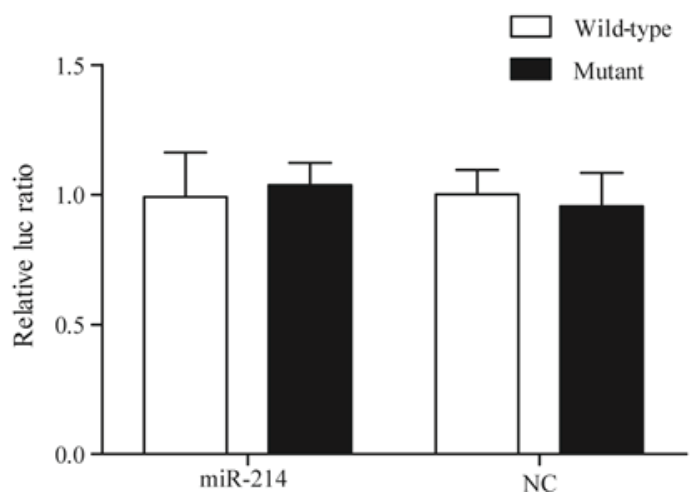

Figure 3. TLR5 is a candidate target gene of miR-214. Computational analysis and luciferase assay were conducted to examine the underlying mechanism of action of miR-214 in sepsis. (A) Schematic comparison of the 'seed sequence' in the 3'-UTR of TLR5 and miR-214. (B) The luciferase activity of the wild-type, and not the mutant, TLR5 3'-UTR was reduced in miR-214 overexpressing cells. (C) Schematic comparison of the 'seed sequence' in the 3'-UTR of TRAF3 and miR-214. (D) miR-214 had no obvious effect on the luciferase activity of the wild-type or mutant TLR5 3'-UTR. "P<0.01 vs. wild-type. UTR, untranslated region; luc, luciferase; miR, microRNA; TLR5, Toll-like receptor 5; TRAF3, TNF receptor-associated factor 3; Mut, mutant; $\mathrm{NC}$, negative control

LPS-treated cells exhibited a lower expression level of miR-214 (Fig. 7A) and higher expression levels of TLR5 mRNA (Fig. 7B) and protein (Fig. 7C), TNF- $\alpha$ (Fig. 7D), IL-6 (Fig. 7E) and IL-10 (Fig. 7F) compared with untreated cells. Furthermore, MALAT1 shRNA and miR-214 induced a further decrease in miR-214 expression compared with the scramble control, and the suppression effects of miR-214 on TLR5, TNF- $\alpha$, IL- 6 and IL-10 expression were further enhanced. Finally, MALAT1 shRNA and miR-214 a induced significant decrease in TLR5 mRNA (Fig. 7B) and protein (Fig. 7C), TNF- $\alpha$ (Fig. 7D), IL-6 (Fig. 7E) and IL-10 (Fig. 7F) expression levels compared with the scramble control, and the inhibitory effects of miR-214 on the expression levels of the above factors appeared to be increased in cells treated with 
A

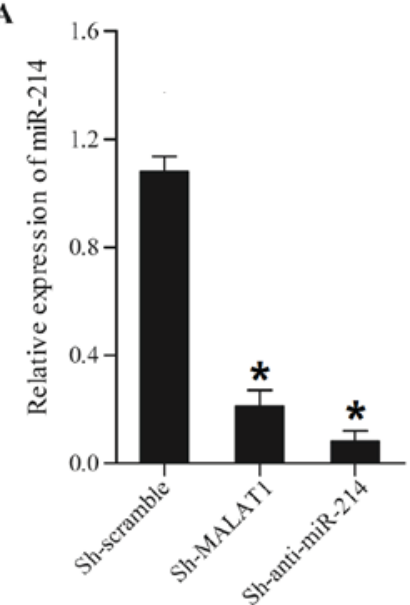

D

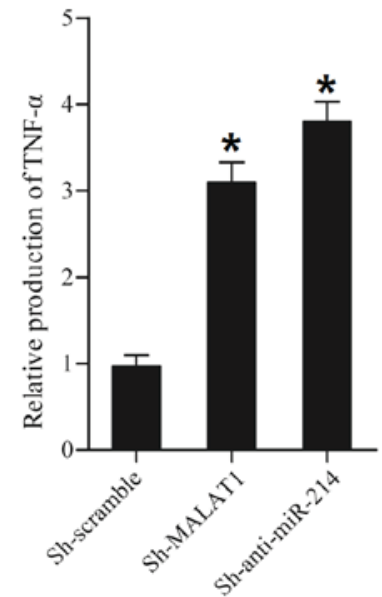

B

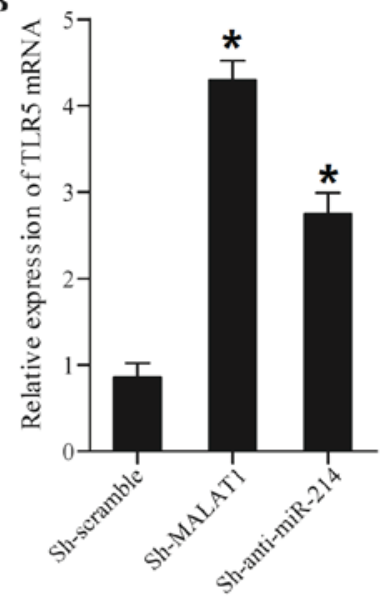

E

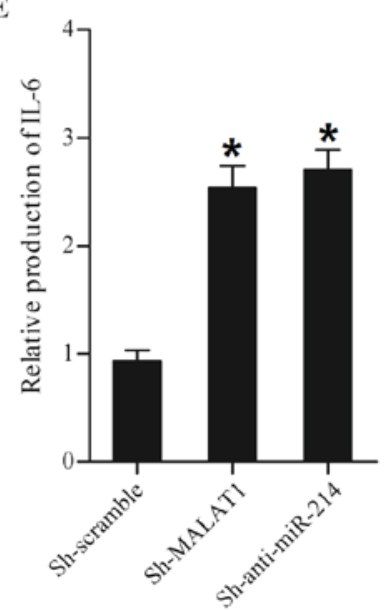

C
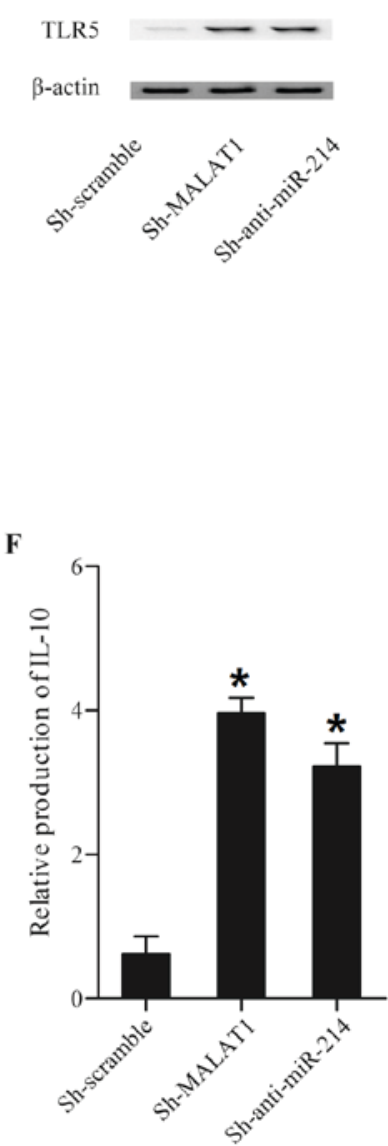

Figure 4. Expression levels of miR-214, TLR5, TNF- $\alpha$, IL-6 and IL-10 in THP-1 cells transfected with Sh-MALAT1 and Sh-anti-miR-214. Expression levels of miR-214, TLR5, TNF- $\alpha$, IL-6 and IL-10 in THP-1 cells transfected with Sh-MALAT1 and Sh-anti-miR-214 were determined using reverse transcription-quantitative polymerase chain reaction, western blot and ELISA analyses. (A) Sh-MALAT1 and Sh-anti-miR-214 downregulated miR-214 expression compared with the scramble control. (B) Sh-MALAT1 and Sh-anti-miR-214 enhanced TLR5 mRNA expression compared with the scramble control. (C) TLR5 protein expression was increased following transfection with constructs containing Sh-MALAT1 and Sh-anti-miR-214 compared with the scramble control. (D) The expression of TNF- $\alpha$ in the Sh-MALAT1 and Sh-anti-miR-214 treatment groups was significantly increased compared with the scramble control. (E) The expression of IL-6 in the Sh-MALAT1 and Sh-anti-miR-214 treatment groups was upregulated compared with the scramble control. (F) The expression level of IL-10 in cells was upregulated subsequent to transfection with Sh-MALAT1 and Sh-anti-miR-214 compared with the scramble control. "P<0.01 vs. respective Sh-scramble group. Sh, short hairpin; MALAT1, metastasis-associated lung adenocarcinoma transcript-1; miR, microRNA; TLR5, Toll-like receptor 5; TNF- $\alpha$, tumor necrosis factor- $\alpha$; IL, interleukin.

LPS compared with those in cells not treated with LPS. The results collectively suggested that MALAT1 promoted TLR5, TNF- $\alpha$, IL- 6 and IL-10 expression by inhibiting miR-214.

\section{Discussion}

The discovery of MALAT1, and other lncRNAs including p50-associated COX-2 extragenic RNA, Lethe and nuclear factor (NF) $-\kappa B$ interacting lncRNA, as novel $N F-\kappa B$ modulators, has added complexity to the understanding of the regulation of this crucial transcription factor, which is important in inflammation and immunity (20). It has been demonstrated that MALAT1 is necessary for the close control of inflammatory reactions triggered by LPS, revealing the potential role of MALAT1 in modulating inflammation and innate immunity (20). Recently, a report identified that the glucose-mediated increase in the inflammatory mediators IL- 6 and TNF- $\alpha$ is modulated by MALAT1 via stimulation of SAA3 (13). It has been reported that MALAT1, which was initially regarded as a prognostic marker for non-small cell lung cancer, may serve a role in regulating the expression of inflammatory mediators (20). As a highly conserved long non-coding RNA, in addition to nuclear-enriched transcript 2, MALAT1 is deregulated in a range of cancer types. MALAT1 was identified as a prognostic marker for lung cancer metastasis, and has also has been associated with a number of other human cancer types (11). MALAT1 functions as an oncogene in esophageal squamous cell carcinoma (ESCC), and it modulates ESCC growth by adjusting the ataxia telangiectasia mutated serine/threonine kinase-checkpoint kinase 2 pathway (21). In the present study, it was identified that burn injury decreased the white blood cell count, and the suppressive effect of post-burn infection on WBCs was much stronger, although this was eliminated by shMALAT1. Burn injury up-regulated MALAT1, TLR5, TNF- $\alpha$, IL-6 and IL-10 expression levels, while the suppressive effects of post-burn infection on TNF- $\alpha$, IL-6 and IL-10 expression levels were stronger, and were abrogated by shMALAT1. Furthermore, 
A
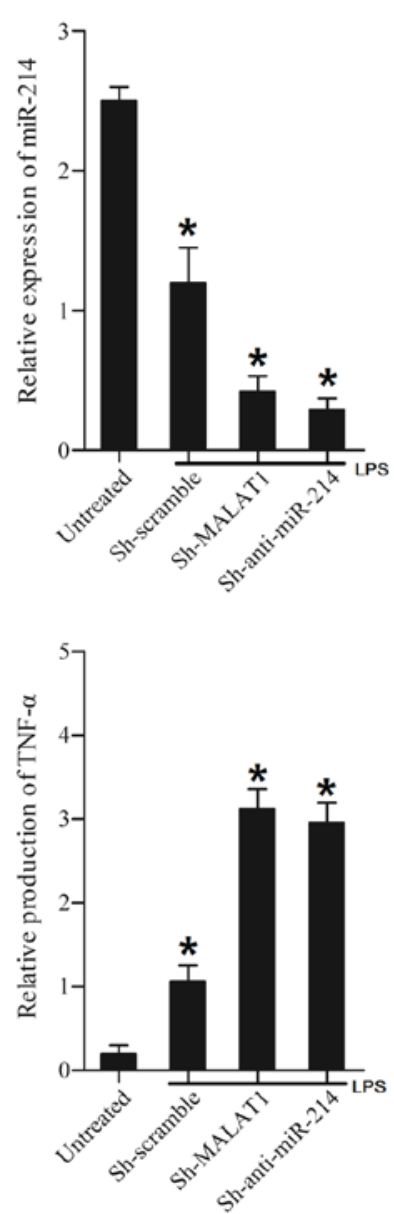

B

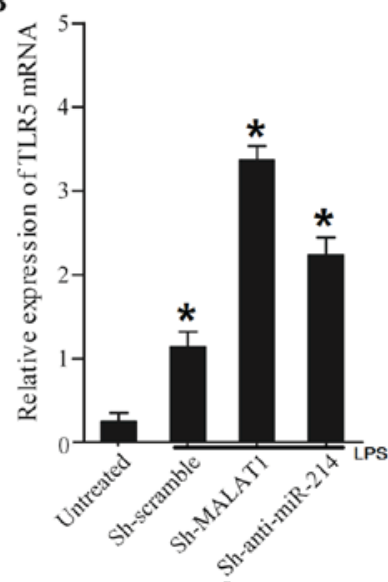

$\mathbf{E}$

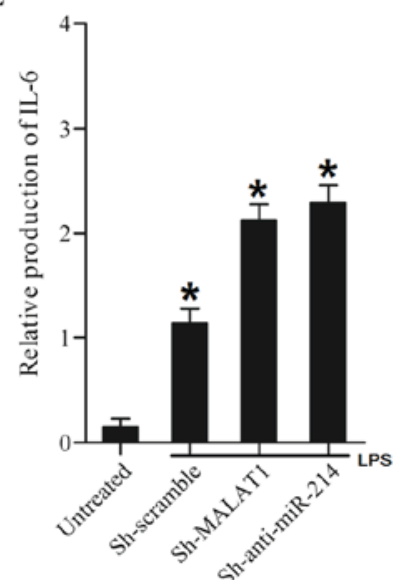

C

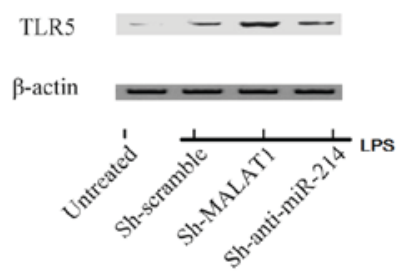

$\mathbf{F}$

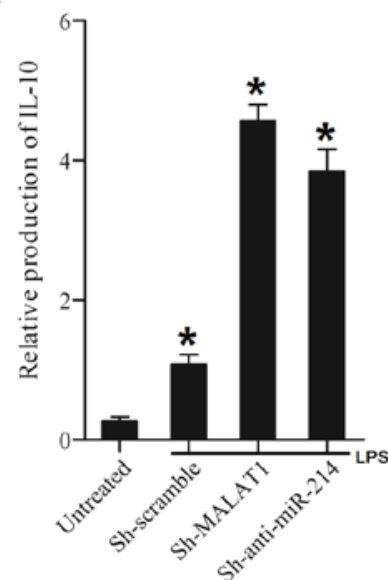

Figure 5. Treatment of LPS altered expression levels of miR-214, TLR5, TNF- $\alpha$, IL-6 and IL-10 in THP-1 cells or LPS-treated cells transfected with Sh-MALAT1, Sh-anti-miR-214. Expression levels of miR-214,TLR5, TNF- $\alpha$,IL-6 and IL-10 in THP-1 cells or LPS-treated cells transfected with Sh-MALAT1, Sh-anti-miR-214 were determined using reverse transcription-quantitative polymerase chain reaction, western blot and ELISA analyses. (A) Treatment with LPS decreased miR-214 expression compared with untreated cells. The miR-214 expression level in LPS-treated cells transfected with Sh-MALAT1 or Sh-anti-miR-214 were significantly decreased compared with the scramble control, and this effect was more marked with Sh-anti-miR-214. (B) LPS-treated cells displayed a higher level of TLR5 mRNA compared with untreated cells. TLR5 mRNA expression levels in LPS-treated cells transfected with Sh-MALAT1 or Sh-anti-miR-214 were significantly increased compared with the scramble control, and this effect was more marked with Sh-anti-miR-214. (C) LPS increased the TLR5 protein expression level compared with that in untreated cells. TLR5 protein expression in LPS-treated cells transfected with Sh-MALAT1 and Sh-anti-miR-214 were further increased. (D) TNF- $\alpha$ expression in LPS-treated cells was significantly increased compared with LPS-untreated cells, while TNF- $\alpha$ expression in LPS-treated cells transfected with Sh-MALAT1 and Sh-anti-miR-214 were further increased compared with LPS-treated cells. (E) LPS-treated cells exhibited a higher level of IL-6 compared with LPS-untreated cells, while IL-6 expression in LPS-treated cells transfected with Sh-MALAT1 and Sh-anti-miR-214 was further increased compared with LPS-treated cells. (F) IL-10 expression in LPS-treated cells was significantly increased compared with LPS-untreated cells, while IL-10 expression in LPS-treated cells transfected with Sh-MALAT1 and Sh-anti-miR-214 was further increased compared with LPS-treated cells.

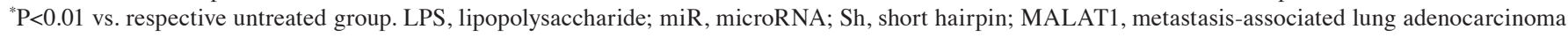
transcript-1; TLR5, Toll-like receptor 5; TNF- $\alpha$, tumor necrosis factor- $\alpha$; IL, interleukin.

the present study investigated whether MALAT1, miR-214 and TLR5 were associated with burn injury and post-burn sepsis, and revealed that MALAT1 promoted burn severity by inhibiting miR-214 production and further enhancing TLR5 expression.

Previous reports have demonstrated the role of miR-214 in inflammatory responses in vitro. Li et al (22) demonstrated that miR-214 is markedly increased in human monocytes and THP- 1 cells following treatment with advanced glycation end products, compromises phosphatase and tensin homolog (PTEN) expression and postpones the apoptosis of THP-1 cells. Jindra et al (23) demonstrated that miR-214 is also markedly elevated in active T cells and is important for enhancing the proliferation and activation of $\mathrm{T}$ cells by affecting PTEN. It has been indicated that overexpression of
miR-214 markedly enhances IL- 6 and TNF- $\alpha$ expression in bone marrow derived macrophages (24). Gonzales et al (25) reported that miR-214 expression was substantially increased, which is consistent with our findings, in an inflammatory macrophage model. Increasing evidence demonstrates that a large portion of IncRNAs are able to function as miRNA sponges by sharing common miRNA response elements, impacting regulation by suppressing available miRNA activity at the post-transcriptional level. It has been demonstrated the rs619586A $>$ G single-nucleotide polymorphism is able to trigger the capture of miR-214 by MALAT1 (15). It has also been revealed that the miR-214 directly targets MALAT1 with the rs619586G allele and inhibits the expression of MALAT1, and miR-214 inhibitors reverse this inhibitory effect; miR-214 inhibitors may release the 
A

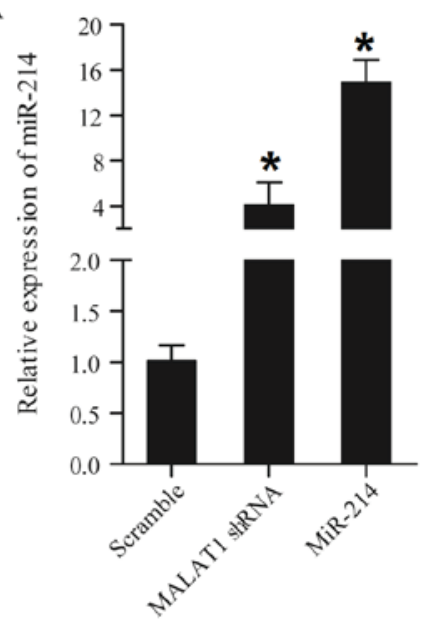

D

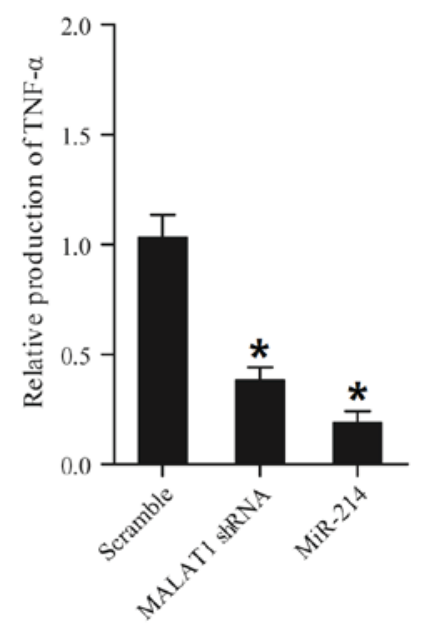

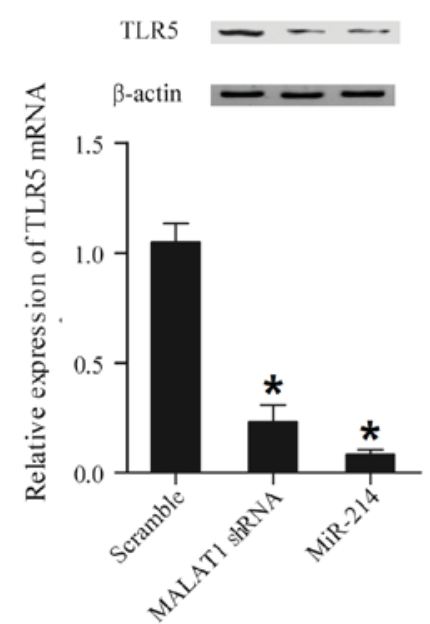

$\mathbf{E}$

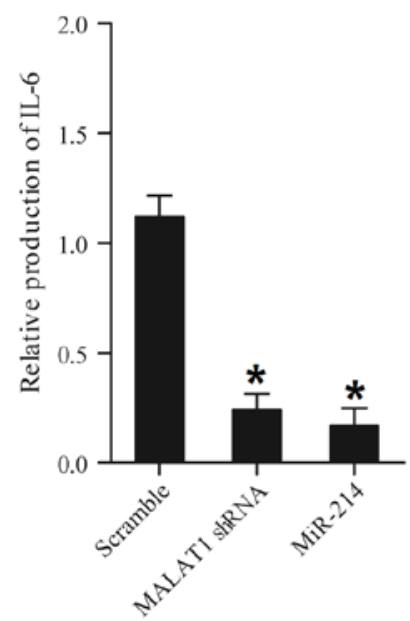

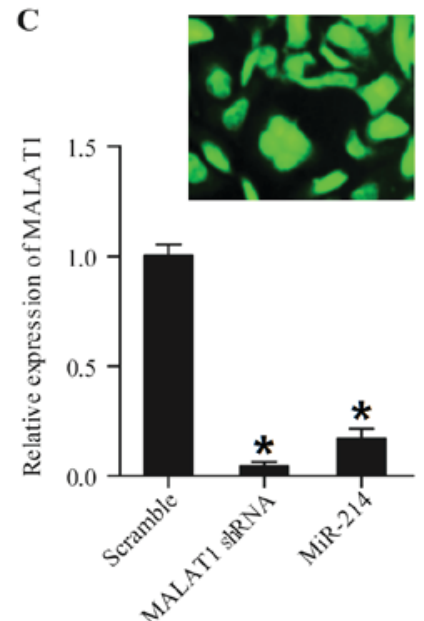

F

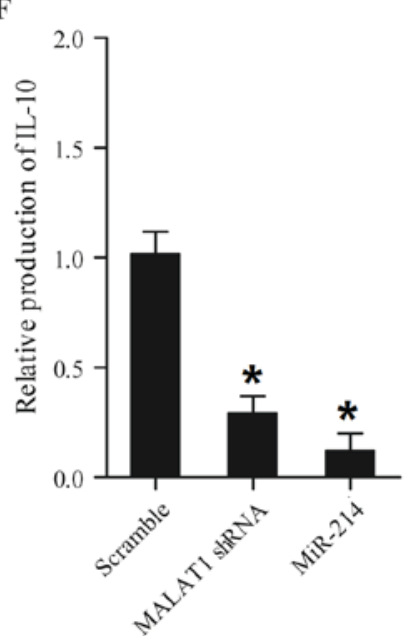

Figure 6. Expression levels of miR-214, TLR5, TNF- $\alpha$, IL-6 and IL-10 in mouse peripheral blood mononuclear cells transfected with MALAT1 shRNA and miR-214. Expression levels of miR-214, TLR5, TNF- $\alpha$, IL-6 and IL-10 in mouse peripheral blood mononuclear cells transfected with MALAT1 shRNA and miR-214 were determined using reverse transcription-quantitative polymerase chain reaction, western blot and ELISA analyses. (A) shRNA and miR-214 enhanced miR-214 expression compared with the scramble control, and the effect was strongest with miR-214. (B) TLR5 mRNA expression (lower panel) and protein expression (upper panel) in the MALAT1 shRNA or miR-214 group was increased compared with the scramble control, and the effect was strongest in the miR-214 group. (C) MALAT1 shRNA was successfully transfected into the cells (upper panel) and MALAT1 shRNA and miR-214 reduced MALAT expression (lower panel) compared with the scramble control; this effect was strongest with miR-214 (scale bar: $10 \mu \mathrm{m}$ ). (D) Expression of TNF- $\alpha$ in the MALAT1 shRNA and miR-214 treatment groups was significantly decreased compared with the scramble control, and this effect was strongest in the miR-214 group. (E) Expression of IL-6 in the miR-214 treatment group was slightly decreased compared with the MALAT1 shRNA group, and expression in the two treatment groups was significantly decreased compared with the scramble control. (F) Expression of IL-10 in the MALAT1 shRNA and miR-214 treatment groups significantly decreased compared with the scramble control, and this effect was more marked in the miR-214 group. "P $<0.01$ vs. respective scramble group. miR, microRNA; Sh, short hairpin; MALAT1, metastasis-associated lung adenocarcinoma transcript-1; TLR5, Toll-like receptor 5; TNF- $\alpha$, tumor necrosis factor- $\alpha$; IL, interleukin.

inhibitory effect of endogenous miR-214 on MALAT1 with the rs619586G allele, and hence trigger increased expression of MALAT1 (15). In the present study, it was demonstrated that miR-214 directly targets the TLR5 3'UTR, and miR-214 markedly inhibited the luciferase activity of the wild-type TLR5 3'UTR and had no effect on the luciferase activity of the mutant TLR5 3'UTR.

TLR5 is present on various cell types including mast cells, macrophages, monocytes, neutrophils and epithelial cells, and is the receptor for the bacterial structural protein flagellin (26). Flagellin signaling through TLR5 depends on myeloid differentiation primary response protein 88 and interleukin-1 receptor-associated kinase 1 and subsequent stimulation of the phosphatidylinositol 3-kinase, mitogen activated protein kinase and NF- $\kappa$ B pathways $(27,28)$. Similar to other TLR agonists, flagellin has been demonstrated to trigger dendritic cell maturation and stimulation, thereby enhancing lymphocyte movement to secondary lymphoid sites $(29,30)$. Others have demonstrated that spontaneous neutrophil apoptosis is delayed by flagellin via mediation of induced myeloid leukemia cell differentiation protein Mcl-1 and suppression of caspase 3 (31). The present study detected the expression levels of miR-214, TLR5, TNF- $\alpha$, IL-6 and IL-10 in cells transfected with Sh-MALAT1, Sh-anti-miR-214 and miR-214 using RT-qPCR, western blotting and ELISA, and observed that MALAT1 and anti-miR-214 inhibited miR-214 expression while enhancing TLR5, TNF- $\alpha$, IL-6 and IL-10 expression in LPS-untreated or treated cells. MALAT1 shRNA and miR-214 upregulated miR-214 expression while inhibiting TLR5, TNF- $\alpha$, IL-6 and IL-10 expression in LPS-untreated or treated cells. It was also 
A

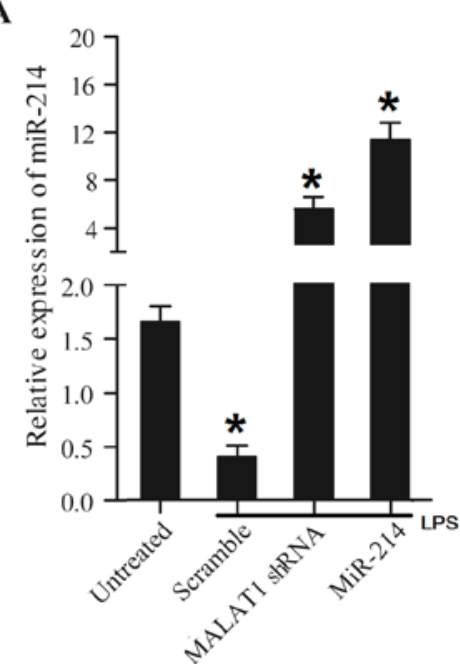

D

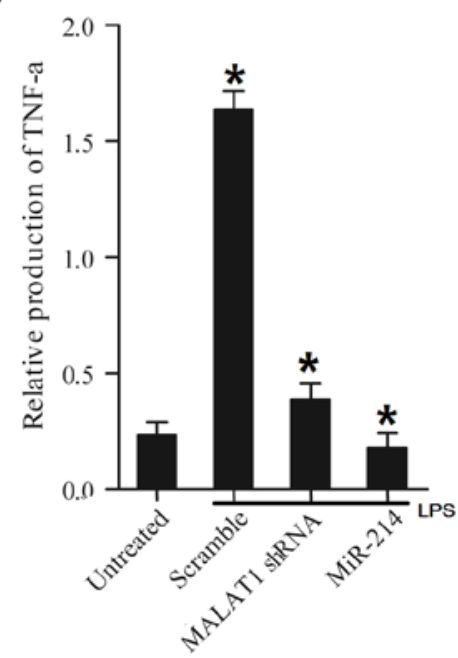

B

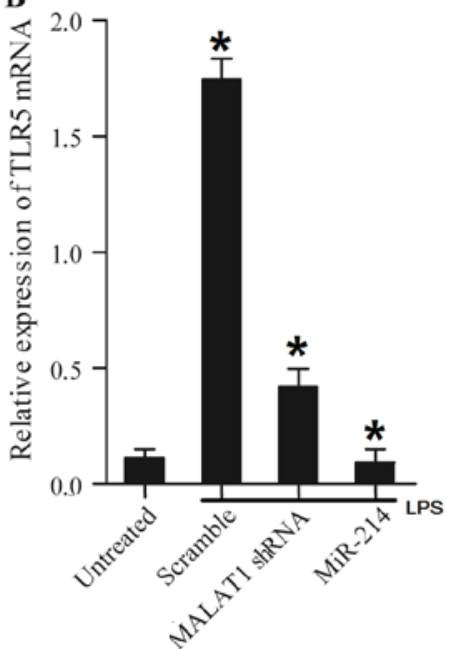

E

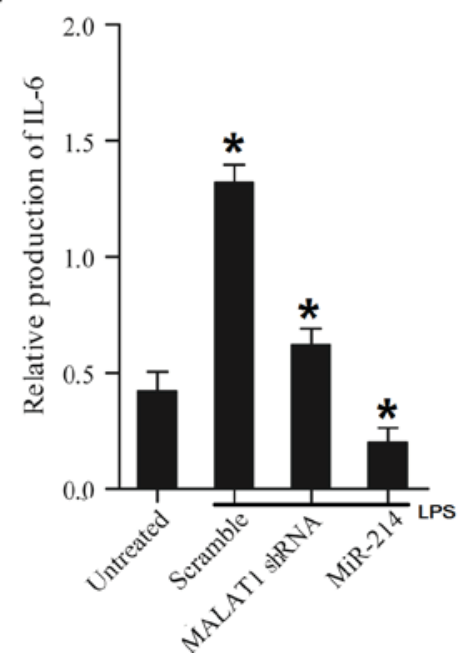

C

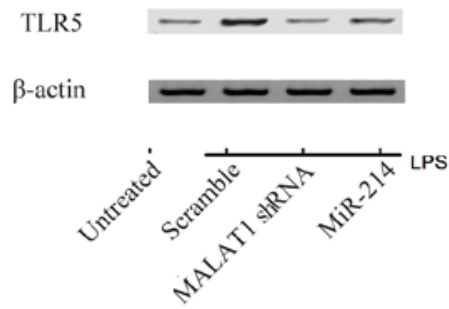

F

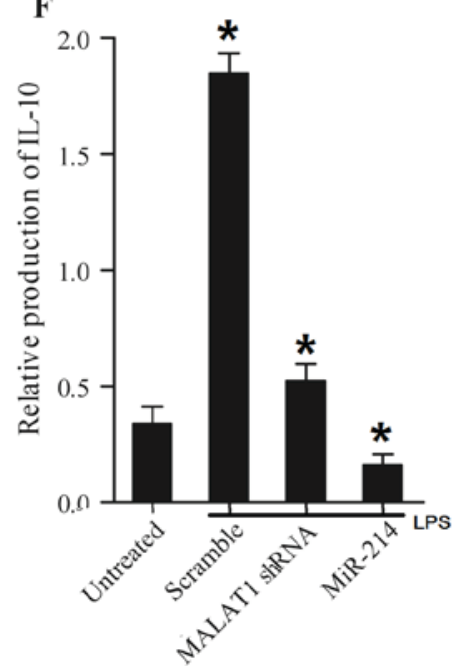

Figure 7. Treatment of LPS altered expression levels of miR-214, TLR5, TNF- $\alpha$, IL-6 and IL-10 in mouse peripheral blood mononuclear cells transfected with MALAT1 shRNA and miR-214. Levels of miR-214, TLR5, TNF- $\alpha$, IL-6 and IL-10 in mouse PBMC cells or LPS-treated cell transfected with MALAT1 shRNA and miR-214 were determined using reverse transcription-quantitative polymerase chain reaction, western blot and ELISA analyses. (A) LPS downregulated miR-214 expression compared with LPS untreated cells. miR-214 expression levels in LPS-treated cells transfected with MALAT1 shRNA and miR-214 were increased compared with the scramble control cells. (B) LPS increased TLR5 mRNA expression compared with cells not treated with LPS, while the TLR5 mRNA expression level in LPS-treated cells decreased following transfection with MALAT1 shRNA and miR-214 compared with the scramble group. (C) TLR5 protein expression levels in LPS-treated cells transfected with MALAT1 shRNA and miR-214 were decreased compared with the scramble group. (D) LPS-treated cells displayed an increased level of TNF- $\alpha$ compared with LPS-untreated cells, while TNF- $\alpha$ expression levels in LPS-treated cells transfected with MALAT1 shRNA and miR-214 were inhibited compared with the scramble group. TNF- $\alpha$ expression the in miR-214 group was further decreased compared with the MALAT1 shRNA group. (E) LPS-treated cells exhibited a higher level of IL-6 compared with LPS-untreated cells, while IL-6 expression in LPS-treated cells transfected with MALAT1 shRNA and miR-214 was notably reduced compared with the scramble group. The effect of miR-214 was stronger compared with that of MALAT1 shRNA. (F) IL-10 expression in LPS-treated cells was significantly increased compared with LPS-untreated cells, while IL-10 expression in LPS-treated cells transfected with MALAT1 shRNA and miR-214 was repressed compared with the scramble group. This effect was more marked in the miR-214 group compared with the MALAT1 shRNA group. *P<0.01 vs. respective untreated group. LPS, lipopolysaccharide; miR, microRNA; Sh, short hairpin; MALAT1, metastasis-associated lung adenocarcinoma transcript-1; TLR5, Toll-like receptor 5; TNF- $\alpha$, tumor necrosis factor- $\alpha$; IL, interleukin.

revealed that LPS caused an evident decrease in miR-214 expression, while inducing a marked increase on TLR5, TNF- $\alpha$, IL- 6 and IL-10 levels. Sepsis has been referred to a systemic inflammation syndrome induced by infection (32). It is currently considered that the majority of patients who succumb to sepsis have unaddressed immune inhibition septic foci (33). Complex mechanisms have been implicated in cell signaling and bacterial sensing, which are regulated during sepsis (34). Immunity to bacterial infection is induced when pattern recognition receptors on phagocytes, including TLRs, identify the pathogen-associated molecular patters produced by the infecting microorganism (35). TLR5 detects bacterial flagellin. It is produced in various cell types, including epithelial cells, dendritic cells, mastocytes and monocytes (35). For instance, in a model of spontaneous colitis, TLR5-depleted mice exhibit more severe colitis (36). The production is TLR5 was elevated among septic patients compared with healthy subjects; additionally, TLR5 production was reported to be reduced in septic patients after 7 days at follow-up (14). As for TLR production, TLR5 was elevated in neutrophils and monocytes from septic patients and TLR4 and TLR2 were reduced in neutrophils in the surviving patients at follow-up (14). 
There are certain limitations to this study: i) The experiments were only performed in animals and cultured cells, thus further study on human subject is required to confirm the results; and ii) the spectrum of inflammatory cytokines has not been comprehensively studied, and thus certain cytokines, including NF- $\kappa \mathrm{B}$ and its associated components, should be considered in future studies.

In conclusion, the results of the present study demonstrated the association between the dysregulation of MALAT1/miR-214/TLR5 and the risk of post-burn sepsis.

\section{Acknowledgements}

Not applicable.

\section{Funding}

No funding was received.

\section{Availability of data and materials}

The datasets used and/or analyzed during the present study are available from the corresponding author on reasonable request.

\section{Authors' contributions}

FG made substantial contributions to the design of the present study, analyzed the data and reviewed the manuscript. RC collected the data and prepared the manuscript; YX made substantial contributions to the acquisition and analysis of data; QZ made substantial contributions to the collected of data and literature, and prepared the manuscript; HG made substantial contributions to the design of the present study and reviewed the manuscript. All authors read and approved the final manuscript.

\section{Ethics approval and consent to participate}

The Institutional Animal Care and Use Committee of Baoji Center Hospital (Baoji, China) approved all procedures involving animals.

\section{Patient consent for publication}

Not applicable.

\section{Competing interests}

The authors declare that they have no competing interests.

\section{References}

1. Murray CK: Infections in burns: J Trauma 62: S73, 2007.

2. Pruitt BA Jr, McManus AT, Kim SH and Goodwin CW: Burn wound infections: Current status. World J Surg 22: 135-145, 1998.

3. Appelgren P, Bjornhagen V, Bragderyd K, Jonsson CE and Ransjo U: A prospective study of infections in burn patients. Burns 28: 39-46, 2002.

4. Branski LK, Al-Mousawi A, Rivero H, Jeschke MG, Sanford AP and Herndon DN: Emerging infections in burns. Surgical Infections 10: 389-397, 2009.

5. Williams FN, Herndon DN, Hawkins HK, Lee JO, Cox RA, Kulp GA, Finnerty CC, Chinkes DL and Jeschke MG: The leading causes of death after burn injury in a single pediatric burn center. Crit Care 13: R183, 2009.
6. Mann EA, Baun MM, Meininger JC and Wade CE: Comparison of mortality associated with sepsis in the burn, trauma, and general intensive care unit patient: A systematic review of the literature. Shock 37: 4-16, 2012.

7. Carninci P, Kasukawa T, Katayama S, Gough J, Frith MC Maeda N, Oyama R, Ravasi T, Lenhard B, Wells C, et al; RIKEN Genome Exploration Research Group and Genome Science Group (Genome Network Project Core Group): The transcriptional landscape of the mammalian genome. Science 309: 1559-1563, 2005.

8. Kim VN, Han J and Siomi MC: Biogenesis of small RNAs in animals. Nat Rev Mol Cell Biol 10: 126-139, 2009.

9. Mercer TR, Dinger ME and Mattick JS: Long non-coding RNAs: Insights into functions. Nat Rev Genet 10: 155-159, 2009.

10. Fabian MR, Sonenberg N and Filipowicz W: Regulation of mRNA translation and stability by microRNAs. Annu Rev Biochem 79: 351-379, 2010

11. Gutschner T, Hammerle M and Diederichs S: MALAT1-A paradigm for long noncoding RNA function in cancer. J Mol Med (Berl) 91: 791-801, 2013.

12. Yan B, Tao ZF, Li XM, Zhang H, Yao J and Jiang Q: Aberrant expression of long noncoding RNAs in early diabetic retinopathy. Invest Ophthalmol Vis Sci 55: 941-951, 2014.

13. Puthanveetil P, Chen S, Feng B, Gautam A and Chakrabarti S: Long non-coding RNA MALAT1 regulates hyperglycaemia induced inflammatory process in the endothelial cells. J Cell Mol Med 19: 1418-1425, 2015.

14. Silva SC, Baggio-Zappia GL, Brunialti MK, Assuncao MS, Azevedo LC, Machado FR and Salomao R: Evaluation of toll-like, chemokine, and integrin receptors on monocytes and neutrophils from peripheral blood of septic patients and their correlation with clinical outcomes. Braz J Med Biol Res 47: 384-393, 2014.

15. Zhuo Y, Zeng Q, Zhang P, Li G, Xie Q and Cheng Y: Functional polymorphism of IncRNA MALAT1 contributes to pulmonary arterial hypertension susceptibility in Chinese people. Clin Chem Lab Med 55: 38-46, 2017.

16. Lahiri R, Derwa Y, Bashir Z, Giles E, Torrance HD, Owen HC, O'Dwyer MJ, O'Brien A, Stagg AJ, Bhattacharya S, et al: Systemic inflammatory response syndrome after major abdominal surgery predicted by early upregulation of TLR4 and TLR5. Ann Surg 263: 1028-1037, 2016.

17. Liu Y, Zhou Q, Wang Y, Liu Z, Dong M, Wang Y, Li X and Hu D: Negative pressure wound therapy decreases mortality in a murine model of burn-wound sepsis involving pseudomonas aeruginosa infection. PLoS One 9: e90494, 2014.

18. Livak KJ and Schmittgen TD: Analysis of relative gene expression data using real-time quantitative PCR and the $2-\Delta \Delta \mathrm{Ct}$ method. Methods 25: 402-408, 2001.

19. Iwagaki A, Porro M and Pollack M: Influence of synthetic antiendotoxin peptides on lipopolysaccharide (LPS) recognition and LPS-induced proinflammatory cytokine responses by cells expressing membrane-bound CD14. Infect Immun 68: 1655-1663, 2000.

20. Zhao G, Su Z, Song D, Mao Y and Mao X: The long noncoding RNA MALAT1 regulates the lipopolysaccharide-induced inflammatory response through its interaction with NF-kappaB. FEBS Lett 590: 2884-2895, 2016.

21. Hu L, Wu Y, Tan D, Meng H, Wang K, Bai Y and Yang K: Up-regulation of long noncoding RNA MALAT1 contributes to proliferation and metastasis in esophageal squamous cell carcinoma. J Exp Clin Cancer Res 34: 7, 2015.

22. Li LM, Hou DX, Guo YL, Yang JW, Liu Y, Zhang CY and Zen K: Role of microRNA-214-targeting phosphatase and tensin homolog in advanced glycation end product-induced apoptosis delay in monocytes. J Immunol 186: 2552-2560, 2011.

23. Jindra PT, Bagley J, Godwin JG and Iacomini J: Costimulationdependent expression of microRNA-214 increases the ability of T cells to proliferate by targeting Pten. J Immunol 185: 990-997, 2010.

24. Zhao L, Liu YW, Yang T, Gan L, Yang N, Dai SS and He F: The mutual regulation between miR-214 and A2AR signaling plays an important role in inflammatory response. Cell Signal 27: 2026-2034, 2015

25. Gonzales JN, Gorshkov B, Varn MN,Zemskova MA,Zemskov EA, Sridhar S, Lucas R and Verin AD: Protective effect of adenosine receptors against lipopolysaccharide-induced acute lung injury. Am J Physiol Lung Cell Mol Physiol 306: L497-L507, 2014.

26. Honko AN and Mizel SB: Effects of flagellin on innate and adaptive immunity. Immunol Res 33: 83-101, 2005.

27. Moors MA, Li L and Mizel SB: Activation of interleukin-1 receptor-associated kinase by gram-negative flagellin. Infect Immun 69: 4424-4429, 2001 
28. Gohda J, Matsumura T and Inoue J: Cutting edge: TNFR-associated factor (TRAF) 6 is essential for MyD88-dependent pathway but not toll/IL-1 receptor domain-containing adaptor-inducing IFN-beta (TRIF)-dependent pathway in TLR signaling. J Immunol 173: 2913-2917, 2004.

29. Means TK, Hayashi F, Smith KD, Aderem A and Luster AD: The Toll-like receptor 5 stimulus bacterial flagellin induces maturation and chemokine production in human dendritic cells. J Immunol 170: 5165-5175, 2003.

30. Mizel SB and Bates JT: Flagellin as an adjuvant: Cellular mechanisms and potential. J Immunol 185: 5677-5682, 2010.

31. Salamone GV, Petracca Y, Fuxman Bass JI, Rumbo M, Nahmod KA, Gabelloni ML, Vermeulen ME, Matteo MJ, Geffner JR and Trevani AS: Flagellin delays spontaneous human neutrophil apoptosis. Lab Invest 90: 1049-1059, 2010.

32. Bone RC, Sibbald WJ and Sprung CL: The ACCP-SCCM consensus conference on sepsis and organ failure. Chest 101: 1481-1483, 1992.
33. Boomer JS, To K, Chang KC, Takasu O, Osborne DF, Walton AH, Bricker TL, Jarman SD II, Kreisel D, Krupnick AS, et al: Immunosuppression in patients who die of sepsis and multiple organ failure. JAMA 306: 2594-2605, 2011.

34. Salomao R, Brunialti MK, Rapozo MM, Baggio-Zappia GL, Galanos $\mathrm{C}$ and Freudenberg M: Bacterial sensing, cell signaling, and modulation of the immune response during sepsis. Shock 38: 227-242, 2012

35. Janeway CA Jr and Medzhitov R: Innate immune recognition. Annu Rev Immunol 20: 197-216, 2002.

36. Vijay-Kumar M, Sanders CJ, Taylor RT, Kumar A, Aitken JD, Sitaraman SV, Neish AS, Uematsu S, Akira S, Williams IR and Gewirtz AT: Deletion of TLR5 results in spontaneous colitis in mice. J Clin Invest 117: 3909-3921, 2007. 